\title{
El capital intelectual y la calidad del servicio en la Facultad de Ciencias Empresariales en la Universidad Privada de Tacna
}

\author{
The intellectual capital and the quality of service in the Faculty of Business at the Private \\ University of Tacna
}

\section{Eloyna Lucia Peñaloza Arana ${ }^{1}$}

\section{RESUMEN}

Objetivo: El presente trabajo de investigación se llevó a cabo con el propósito de determinar la Influencia del Capital Intelectual en la calidad del servicio en la Facultad de Ciencias Empresariales en la Universidad Privada de Tacna 2012.

Método: El trabajo corresponde a una investigación de nivel relacional; y el diseño es no experimental, transeccional, ya que se trabajó el período 2012. Para tal propósito se consideró la información obtenida a través de la aplicación del instrumento de medición del cuestionario para evaluar el capital intelectual y la calidad de servicio que se brinda en la Facultad de Ciencias Empresariales, los cuales fueron aplicados a los docentes y alumnos con la técnica de la encuesta, utilizándose la Escala de likert. Finalizada la fase de análisis e interpretación de los resultados se determinó que en la Facultad de Ciencias Empresariales de la Universidad Privada de Tacna, el Capital Intelectual influye significativamente en la calidad de servicio. El modelo de medición consta de 24 indicadores de escala para capital intelectual y 9 para competitividad; y se comprueba que el conjunto de activos del capital intelectual ejercen una influencia positiva en la competitividad.

Resultado: Se han determinado que el capital intelectual evaluado a través de sus dimensiones capital humano, estructural y relacional es adecuado; y la calidad de servicio según dimensiones elementos tangibles, confiabilidad, capacidad de respuesta, seguridad y empatía es percibido como regular por los estudiantes y docentes de la FACEM.

Conclusiones: Preciso enfatizar en el potenciamiento del capital intelectual para poder brindar una mejor calidad de servicio en la Universidad Privada de Tacna.

\section{PALABRAS CLAVE}

Capital intelectual, calidad del servicio, activos intangibles, valoración de las empresas.

\begin{abstract}
Objective: The present research was conducted in order to determine the influence of intellectual capital in the quality of service in the Faculty of Business at the Private University of Tacna 2012.

Method: The work corresponds, whose level corresponds to relational and non-experimental design , transactional, and that it was the period 2012. For this purpose we considered the information obtained through the application of the questionnaire measuring instrument for assessing the intellectual capital and the quality of service provided in FACEM, which were applied to teachers and students with technical survey, using the Likert scale. After the phase of analysis and interpretation of the results was determined that in the Faculty of Business Studies of the Private University of Tacna , intellectual capital significantly influences the quality of service, 2012. The measurement models 24 scale indicators for intellectual capital, and 9 to competitiveness, and it is found that the set of intellectual capital assets have a positive influence on competitiveness.

Result: They have determined that the brainpower evaluated through its human, structural and relational capital size is adequate and the quality of service according to dimensions tangibles, reliability, responsiveness, assurance and empathy is perceived as fair by students and teachers the FACEM.

Conclusions: Definite emphasize the enhancement of intellectual capital to provide better quality of service and thereby convert the FACEM in model school at the Private University of Tacna.
\end{abstract}

\section{KEYWORDS}

Intellectual capital, quality of service, intangible assets, rating of companies.

1. Doctora en Administración, Magister en Administración y Dirección de Empresas, Contador Público Colegiado. Especializada en auditoría, peritaje contable. Actualmente labora como Administradora de la Universidad Privada de Tacna. Tacna - Perú 


\section{Introducción}

El capital intelectual es, a grandes rasgos, el conocimiento adquirido a través de la experiencia de una organización, institución o empresa. Se trata de una información intangible que permitirá, a quien lo gestione adecuadamente aprovechar mejor las oportunidades, dando lugar a la generación de beneficios futuros y el mejoramiento de su calidad de servicio.

Edvinsson fue uno de los primeros en ser consciente de la importancia del capital intelectual dentro de una organización. Presenta el concepto mediante la siguiente metáfora: "una corporación es como un árbol. Hay una parte que es visible (las frutas) y una parte que está oculta (las raíces). Si solamente te preocupas por las frutas, el árbol puede morir. Para que el árbol crezca y continúe dando frutos, será necesario que las raíces estén sanas y nutridas. Esto es válido para las organizaciones: si sólo nos concentramos en los frutos (los resultados) e ignoramos los valores escondidos, la organización no subsistirá en el largo plazo".

De esta forma, este promotor del desarrollo del capital intelectual afirma que "gran parte del valor de una empresa es inexplicable e incontable. El conocimiento, la marca, innovación y otros activos invisibles concentran más creación de valor que los factores clásicos de producción en la gran mayoría de las organizaciones".

Steward, T. A. (1998) por su parte, lo define como "conocimiento, información, propiedad intelectual, experiencia, que puede utilizarse para crear valor. Es fuerza cerebral colectiva. Es difícil de identificar y aún más de distribuir eficazmente. Pero quien lo encuentra y lo explota, triunfa". Steward, T.A. (1998) afirma, además, que "en la nueva era, la riqueza es producto del conocimiento. Éste y la información se han convertido en las materias primas fundamentales de la economía y sus productos más importantes".

De la misma forma, Skandia, A.F.S. (1994)(compañía escandinava de seguros y servicios financieros), define el "capital intelectual como la posesión de conocimientos, experiencia aplicada, tecnología organizacional, relaciones con clientes y destrezas profesionales que dan a la empresa una ventaja competitiva en el mercado".

En resumen, podemos decir que el conocimientos de las personas, sus experiencias, el "saber hacer" de la organización, la satisfacción de los clientes, etc., son activos que explican buena parte de la valoración que el mercado concede a una organización y que, sin embargo, no son recogidos en el valor contable de la misma.

Las Organizaciones del presente, se caracterizan entre otros aspectos por emplear el Capital Intelectual, es decir, el material intelectual, conocimiento, información, propiedad intelectual, experiencia, que puede utilizarse para crear valor, la fuerza cerebral colectiva de la organización, en él se recogen aquellos activos intangibles de la empresa, no reflejados en los estados financieros, pero que contribuyen a la creación de valor organizativo como su mayor ventaja competitiva, en este periodo tan dinámico y cambiante en que se mueven las organizaciones. 
La excelencia del desempeño solo se logrará utilizando los activos intelectuales. No existe otra manera de generar el tipo de desempeño que requerirá una organización para triunfar en la nueva realidad.

\section{Materiales y métodos}

Es una investigación relacional, porque se ocupa de medir la relación de las variables en estudio. El diseño es no experimental (ex pos facto), de corte transversal, ya que el recojo de información se hizo en un solo momento del tiempo, haciendo referencia a un período específico en la Facultad de Ciencias Empresariales de la Universidad Privada de Tacna.

La población son los docentes y estudiantes de la Facultad de Ciencias Empresariales; y se ha determinado el tamaño de la muestra de acuerdo a los criterios matemáticos.

Para la recolección de datos se empleó el instrumento de medición del cuestionario aplicado bajo la técnica de la encuesta el cual fue aplicado para la medición de las variables a estudio: capital intelectual y calidad de servicio. Dicho instrumento de medición fue distribuido a los docentes y estudiantes de la FACEM, los días 19 al 22 de agosto del 2012.

\section{Resultados}

Los conocimientos de las personas clave de la empresa, la satisfacción de los empleados, el know-how, la satisfacción de los clientes, etc., son activos que explican buena parte de la valoración que el mercado concede a una organización y que, sin embargo, no son recogidos en el valor contable de la misma.

En conocimiento de los antecedentes; la presente investigación tiene por objetivo específico determinar la influencia del capital intelectual en la calidad del servicio en la Facultad de Ciencias Empresariales en la Universidad Privada de Tacna; aplicando un cuestionario de capital intelectual a las docentes de la FACEM UPT; en el cual se propusieron 50 ítems elaborados en base a las dimensiones y los indicadores de las variables.

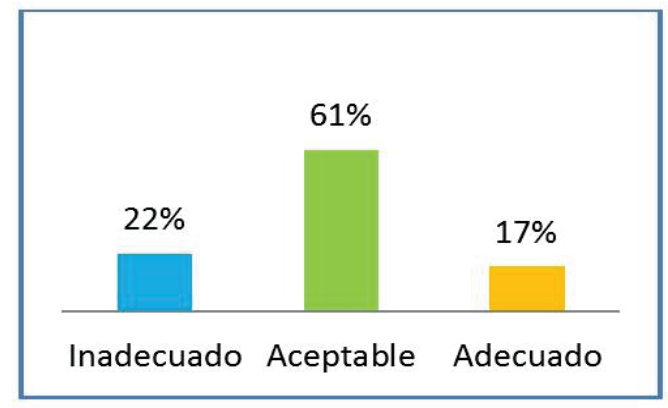

Figura 1.

Capital humano

Del análisis se concluye que el capital humano (conocimientos/educación, habilidades, experiencia y motivación) son aceptables; es preciso indicar que los docentes se destacan por su nivel de capacitación, experiencia y motivación hacia su trabajo (Figura 1).

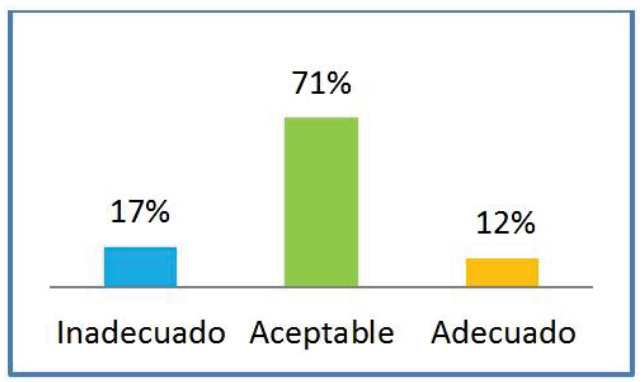

Figura 2.

Capital estructural 
Del análisis se concluye que el capital estructural, según indicadores: tecnología, cultura/valores, estructura, sistemas y procesos, es aceptable; denotándose que en la FACEM los gestores muestran preocupación por dotar de equipos modernos para el desarrollo del proceso enseñanza aprendizaje (Figura 2).

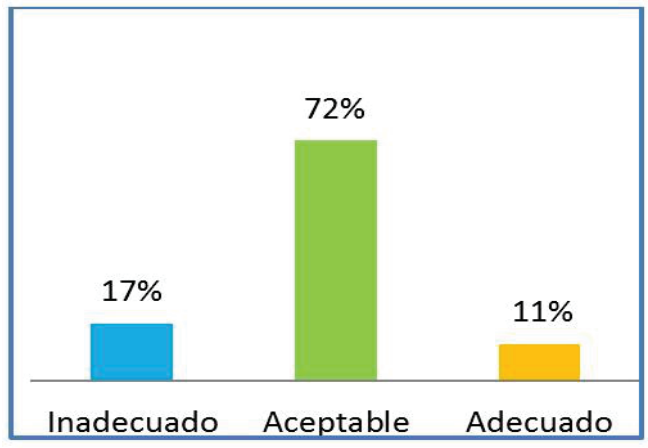

Figura 3.

Capital relacional

Del análisis se concluye que el capital relacional según indicadores: relación con usuarios, proveedores y alianzas formadas son aceptables; denotándose que en la FACEM los gestores muestran preocupación por mantenerse buenas relaciones con los diferentes grupos de interés (Figura 3).

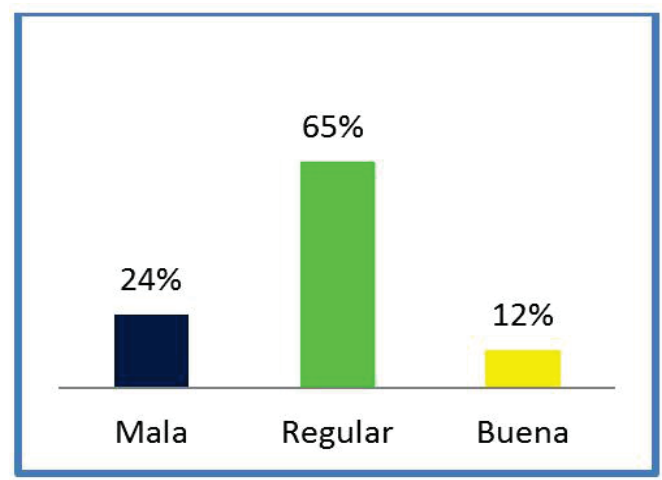

Figura 4.

Capital tangible

El resultado del análisis permite inferir que la calidad de Servicio educativo según elementos tangibles (apariencia de las instalaciones físicas, equipos, personal y materiales de comunicación) son evaluados como regulares por los usuarios (docentes y alumnos); incidiendo en el aspecto de insuficiencia en los materiales y equipos didácticos en la FACEM (Figura 4).

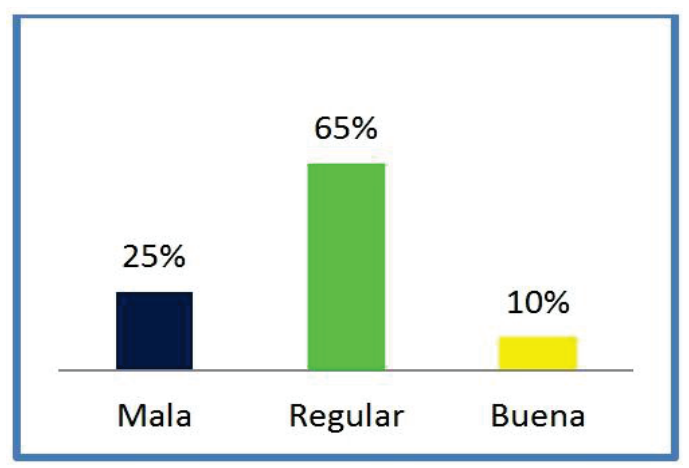

Figura 5.

Capital confiabilidad

El resultado del análisis permite concluir que la calidad de Servicio educativo según confiabilidad (habilidad para ejecutar el servicio prometido de forma fiable y cuidadosa) son evaluados como regulares por los usuarios (docentes y alumnos); incidiendo en el aspecto de capacidad para realizar el servicio haciendo frente a imprevistos y dificultades. (Figura 5).

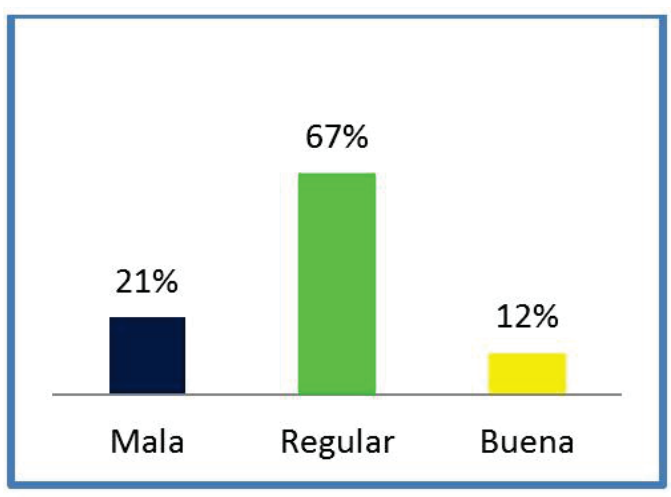

Figura 6.

Capital de respuesta

El resultado del análisis permite concluir que la calidad de Servicio 
educativo según capacidad de respuesta (disposición y voluntad de los trabajadores para ayudar al usuario y proporcionar el servicio), es evaluada como regular por los usuarios (docentes y alumnos); incidiendo en el aspecto de oportunidad de cumplimiento en el servicio requerido. (Figura 6).

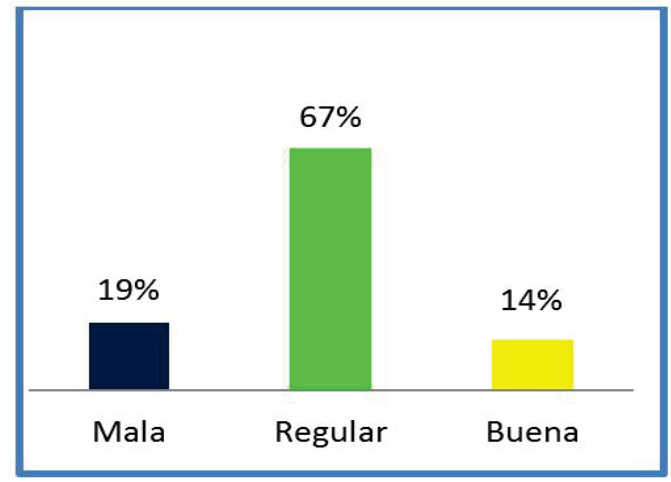

Figura 7.

Seguridad

El resultado del análisis permite concluir que la calidad de Servicio educativo según seguridad (conocimiento y atención, mostrados por los empleados y sus habilidades para inspirar credibilidad y confianza.) es evaluada como regular por los usuarios (docentes y alumnos); incidiendo en el aspecto de amabilidad, igualdad y confianza ante el servicio requerido. (Figura 7 ).

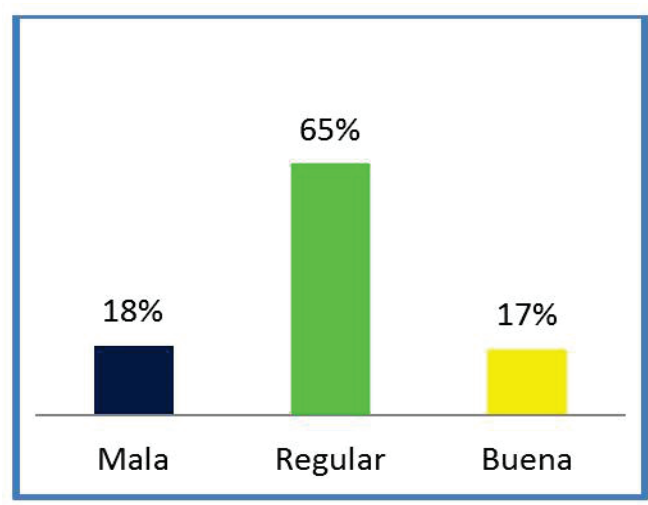

Figura 8.

Empatía
El resultado del análisis permite concluir que la calidad de Servicio educativo según la empatía mostrada (atención Individualizada que se ofrece a los usuarios) es evaluada como regulares por los usuarios (docentes y alumnos); incidiendo en el aspecto de atención individualizada y entendimiento en la satisfacción de necesidades específicas de los usuarios. (Figura 8).

\section{Discusión}

La presente investigación determinó la influencia del capital intelectual en la calidad del servicio en la Facultad de Ciencias Empresariales en la Universidad Privada de Tacna; ya que se comprobó que en esta Facultad, el capital intelectual influye significativamente en la calidad de servicio.

Administrar el capital intelectualencontrarlo, cultivarlo, almacenarlo, compartirlo y venderlo - se está convirtiendo en una de las actividades más importantes para cualquier organización. No es una tarea fácil, puesto que en su mayoría es intangible - no se pueden tocar, mover, o valorar fácilmente.

El libro de Stewart, T.A. (1998) ayuda a clarificar y organizar la gran masa intangible de activos intelectuales con la que cuenta una organización. Comienza por separarlos en tres grupos de capital: humano, estructural y relacional.

Aunque cada uno de ellos sigue siendo intangible, describen cosas que los gestores pueden entender, organizar, administrar y hacer crecer. Más allá de reconocer que la información es el 
activo más importante de la empresa, es necesario traducir las teorías y buenas intenciones en planes $y$ estrategias que lleven a mejoras del desempeño.

En congruencia Arenas, T. \& Lavanderos, L. (2008), en el estudio titulado "Capital intelectual ¿objeto o proceso?: hacia una epistemología del capital intelectual", precisa. Los fundamentos episte-mológicos que mejor interpretan el espíritu del capital intelectual están en la teoría relacional. Esto permite el desarrollo de una nueva perspectiva en relación a la gestión del capital intelectual, la cual debe poner énfasis en como las redes organizan las relaciones a través de una cultura común.

Finalmente profundizando más en esta perspectiva, podemos definir una empresa como un proceso de relaciones, determinado por su cultura y organizado de acuerdo a los intercambios de códigos de agenciamiento y pertenencia entre personas, los cuales orientan el proceso de toma de decisiones hacia la generación de valor. Por tanto el capital intelectual que es conocimiento que genera valor, emerge de la coherencia del proceso relacional entre la estructura de la organización y el proceso decisional de la misma.

Adicionalmente, Román, N. (2004), manifiestan que si empresas u organizaciones de hoy día desean triunfar, deben hacer énfasis en el desarrollo y potenciación de sus activos intangibles (capital intelectual), pues está comprobado que las empresas que actualmente gozan de ventajas competitivas en el mercado son las que han llevado adelante una eficiente gestión de este importante activo.

Así también, Robles, M.; García C. \& Simón, M. G. (2005) señala que la medición y reconocimiento del capital intelectual no es la única barrera a la que tienen que hacer frente las empresas. Su dificultad de gestión aumenta en la medida en que nos encontramos con estructuras organizativas tradicionales caracterizadas por: estructuras burocráticas, liderazgo autoritario, aislamiento del entorno, cultura de ocultación de errores y de información, orientación a la homogeneidad, falta de visión, estratégica, visión a corto plazo, planificación rígida, falta de flexibilidad en las estructuras, individualismo.

En la FACEM de la UPT, se observa que el capital intelectual, medido a través de sus dimensiones capital humano, estructural y relacional son aceptables, los que guardan relación con la calidad de servicio que se brinda a la comunidad universitaria (docentes y alumnos), encontrándose que esta es regular. Es necesario indicar que debido al proceso de Acreditación Universitaria es necesario incidir en este tipo de investigaciones que contribuyan al mejoramiento de la gestión y de esta manera lograr los objetivos y reconocimiento organizacionales.

Los resultados de la presente investigación argumenta que el capital intelectual ha sido evaluado como "adecuado" por los agentes participantes, debido a que se ha encontrado que en porcentajes del 60 , 70 y hasta $80 \%$ han incidido que las fortalezas de la FACEM se encuentran 
en su capital humano, estructural y relacional.

La FACEM de la UPT, cuenta con: personal capacitado, modernas estructuras, y equipos que facilitan el proceso enseñanza aprendizaje, los adecuados elementos del capital intelectual han permitido que la FACEM sea una de las Facultades más modernas, proactivas e innovadoras en la UPT, su plana docente, así como sus alumnos se caracterizan por el desarrollo de habilidades y experiencia que son el motor y permiten el liderazgo de la Facultad en la UPT. Asimismo se observa que la Calidad de Servicio educativo está determinada por elementos tales como: los tangibles, confiabilidad, capacidad de respuesta, seguridad y empatía que repercuten en la gestión institucional.

Por lo tanto se puede afirmar que el Capital Intelectual influye en la Calidad de Servicio en la Facultad de Ciencias Empresariales de la UPT.

\section{Referencias Bibliográficas}

1. Stewart, T.A. (1998). La nueva riqueza de las organizaciones: El capital intelectual. Buenos Aires, Argentina: Ediciones Granica.

2. Skandia, A. F. S. (1994). Visualizing intellectual capital in Skandia. En: $A$ supplement to Skandia's 1994 Annual Report.

3. Arenas, T. \& Lavanderos, L. (2008). Intellectual capital: object or process?. En: Journal of Intellectual Capital, 9 (1), pp. 77-85.
4. Román, N. (2004). Capital Intelectual. En: Revista Visión Gerencial. 2.

5. Robles, M. ; García C. \& Simón, M. G. (2005). El Liderazgo Empresarial en el Siglo XXI y el Capital Intelectual.

E-mail: luciap1100@gmail.com

Recibido: 16/05/14

Aceptado para publicación: 04/06/2014 